\title{
WACOP: PLATAFORMA DE SOFTWARE PARA MONITORAMENTO DO CONSUMO DE ÁGUA RESIDENCIAL DE FORMA INTELIGENTE
}

\author{
Guilherme Silvestre Giazzi, Israel Florentino dos Santos e Leandro Pupo Natale \\ Universidade Presbiteriana Mackenzie \\ Rua da Consolação, 930 - São Paulo, S.P., Brasil
}

\begin{abstract}
RESUMO
Nos dias de hoje, ainda há pessoas que não se preocupam ou dão pouca importância para um assunto tão delicado como a economia de água, seja por não terem essa informação de prontidão ou por não encontrarem facilidade em acompanhá-la. É um dever de todos tratar desse assunto, a fim de que o maior número de indivíduos também faça a sua parte, e a economia de recursos naturais passe a ser uma atividade comum. Sabendo disso, esse artigo apresenta uma solução para ajudar a monitorar o consumo de água com o uso de Internet das Coisas dentro de um contexto de Cidade Inteligente.
\end{abstract}

PALAVRAS-CHAVE

Cidades Inteligentes, IoT, Recursos Naturais, Web 2.0

\section{INTRODUÇÃO}

Desde 2009 a maior parte da população passou a viver em áreas urbanas, fazendo com que a infraestrutura e recursos naturais se tornassem mais escassos (ONU, 2009). Para que todos estejamos preparados para enfrentar uma eminente crise de recursos naturais é necessário mudar nossa forma de ver o mundo, otimizando o uso dos recursos disponíveis, principalmente nas grandes cidades, tornando nossos ambientes urbanos mais inteligentes.

Iniciativas nas dimensões de avaliação de cidades inteligentes propostas por Giffinger et. al (2007) de Economia inteligente, População inteligente, Governança inteligente, Mobilidade inteligente, Meio-ambiente inteligente e Vida inteligente vêm aumentando em diversos lugares do mundo, como na Europa, Estados Unidos, Japão, China, Brasil, Emirados Árabes e Coreia do Sul (Kon e Santana, 2017). Segundo as estatísticas do Fórum de Cidades Inteligentes Chinês, 6 províncias e 51 cidades incluíram Cidades Inteligentes em seu planejamento de trabalho para o governo da China, e, destas, 36 já estão em processo de desenvolvimento (Liu e Peng, 2014).

Neste sentido, este projeto propõe uma plataforma tecnológica para o controle e monitoramento do consumo de água, o WACOP - Water Consumption Platform (Plataforma de Monitoramento do Consumo de Água). Esta plataforma, baseada no gerenciamento de componentes inteligentes conectados à internet e utilizando uma arquitetura de microserviços, permite a coleta, armazenamento, análise e monitoramento do consumo de água potável em diferentes pontos de coletas de uma residência. Os dados coletados são enviados para módulos hospedados em uma nuvem, onde é possível realizar diferentes tipos de análises sobre eles, permitindo, principalmente, uma visão eficiente e detalhada do consumo de água da residência.

\section{REFERENCIAL TEÓRICO}

Tem-se por Cidade Inteligente um centro urbano sustentável e eficiente que oferece alta qualidade de vida para seus habitantes através do gerenciamento ideal de seus recursos (Calvillo et. al, 2016). Tratando-se de gerenciamento inteligente de recursos, Almirall et. al (2013) explica Cidade Inteligente como uma cidade com intensa e avançada tecnologia permitindo a interconexão de pessoas, troca de informações entre os elementos 
da cidade através do uso de tecnologias, a fim de criar uma cidade mais sustentável, mais verde, competitiva, com inovação no comércio, e qualidade de vida melhor.

Este gerenciamento eficiente pode ser obtido através do uso de tecnologias como sensores e dispositivos conectados à internet. Os dados coletados por estes dispositivos podem ser enviados a centros de processamentos de dados, fornecendo informações importantes aos responsáveis pela administração pública. Esta conectividade aliada ao processamento eficiente e inteligente dos dados coletados podem interatuar nas diferentes dimensões de uma cidade inteligente, como economia inteligente, pessoas inteligentes, governo inteligente, mobilidade inteligente, meio ambiente inteligente e vida inteligente (Lombardi et. al, 2012).

Segundo Guillemin e Friess (2009), IoT (IoT - Internet of things; Internet das Coisas) permite que pessoas e coisas se conectem a qualquer momento, de qualquer lugar, com qualquer coisa e qualquer pessoa, idealmente usando qualquer caminho ou rede e qualquer serviço. IoT pressupõe uma rede de objetos físicos interconectados (chamados de "coisas"), incluindo computadores, smart phones, sensores, acionadores, dispositivos em vestimentas, casas, construções, veículos e sistemas de energia. A IoT garante a comunicação de uma grande variedade de tipos de sistemas, aplicações e principalmente sensores, disponibilizando serviços cada vez mais inteligentes, confiáveis e seguros. Desta forma, o coração da implementação de uma cidade inteligente é a interconexão de diversos sensores e dispositivos à Internet e estes dispositivos serem passíveis de envio de dados e recepção de comandos remotos (Mohanty et. al, 2016).

A computação em nuvem está diretamente envolvida, de forma que deve estar pronta para receber todos os dados coletados. A proposta da computação em nuvem é concentrar recursos como hardware e software em algumas localizações físicas e oferecer esses recursos como serviços para uma grande quantidade de consumidores que estão localizados em diferentes regiões geográficas no mundo através da internet por uma maneira eficiente (Perera et. al, 2014).

De acordo com Patidar et. al (2012), os serviços da computação em nuvem podem ser divididos em três camadas principais: Infrastructure as a Service (IaaS), Platfrom as a Service (PaaS). e Software as a Service (SaaS). De uma maneira abrangente, IaaS oferece recursos computacionais como processamento e armazenamento. PaaS é designado para desenvolvedores de software, a fim de permiti-los escrever suas aplicações segundo as especificações de uma plataforma em particular sem precisar se preocupar com o hardware por trás de tudo. Por fim, SaaS é a camada mais visível da computação em nuvem para usuários finais, já que se trata das aplicações em si que são acessadas e usadas. Este projeto adotará a camada PaaS, pois toda a infraestrutura em nuvem disposta está sob responsabilidade dos provedores dos serviços, permitindo que o desenvolvedor apenas utilize esses serviços como repositório para seus dados.

Através do uso destes tipos de sensores é possível criar monitoramentos de recursos utilizados nas cidades de forma a otimizar e incentivar o consumo consciente dos recursos naturais, como o consumo de água potável nas residências e conjuntos habitacionais de uma cidade. Um estudo similar ao deste projeto foi realizado para não apenas coletar o consumo, como também para gerar energia através do fluxo de água e armazenar essas informações com o auxílio do Apache Spark (Domoney et. al, 2015). A principal diferença entre os projetos está no foco abordado por Domoney (2015) na geração e utilização de um recurso através de outro, sendo que o fluxo de água gera energia elétrica. $\mathrm{O}$ projeto apresentado neste artigo propõe o monitoramento inteligente de água em residências, analisando o histórico e criando alertas de consumo de forma a dar mais transparência no uso deste recurso escasso.

Segundo o Procon-SP (órgão governamental do Estado de São Paulo, Brasil, responsável por elaborar e executar políticas públicas de defesa do consumidor), o consumo de água encanada e tratada, per capita sugerida, deve ser em torno de $5,4 \mathrm{~m}^{3}$ por mês. Além do monitoramento, a correta análise permite criar um controle eficiente do consumo e prever com maior precisão o aumento ou diminuição do consumo de água, e assim, possibilitar diferentes ações em diferentes níveis. Por exemplo, em um nível governamental permite a definição de campanhas, políticas públicas, ajustes em investimentos relacionados ao consumo, já em um nível residencial, é possível controlar os gastos. Por fim, este controle permite estimular o consumo de água de forma eficiente com o objetivo de preservar os recursos naturais do planeta.

\section{ARQUITETURA PROPOSTA}

Este artigo apresenta uma proposta de plataforma sistêmica para o monitoramento eficiente do consumo de água em residências. Esta plataforma utiliza como base a componentização e microserviços de forma a permitir um alto grau de escalabilidade e integração com diferentes tipos de sensores e ferramentas, conforme apresentando na Figura 1. 


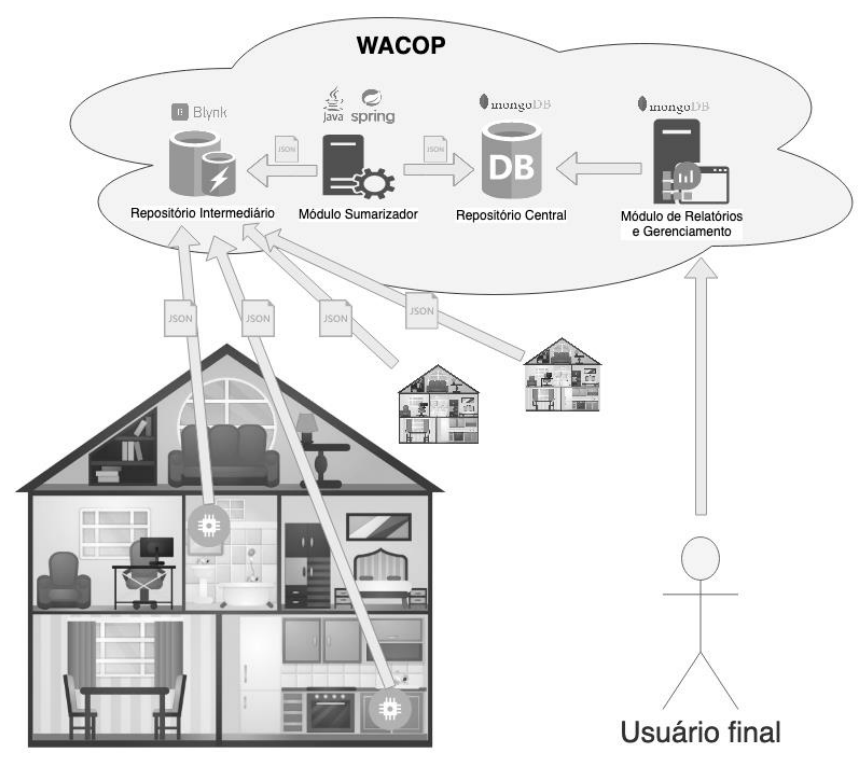

Figura 1. Diagrama apresentando a arquitetura proposta para a Plataforma WACOP

Esta plataforma utiliza um sensor de fluxo de água do tipo SAIER SEN-HZ21WA para coletar o fluxo existente em diferentes saídas de água em cômodos de uma residência, como torneiras e chuveiros. Este sensor deve estar conectado a um Arduino UNO responsável por receber e armazenar os dados coletados temporariamente e enviá-los, via protocolo HTTP, através de uma placa ESP-8266, para um Repositório Intermediário em nuvem. Este repositório de dados é responsável por gerenciar os dados recebidos de todos os sensores de fluxo de água utilizados. Como implementação deste repositório em nuvem a primeira versão implementada da Plataforma WACOP utilizou a plataforma, também em nuvem, Blynk ${ }^{1}$.

Utilizando as bibliotecas do Blynk, é realizada uma requisição POST da média de fluxo de água identificado pelo sensor a cada minuto. Como este tipo de sensor de fluxo de água, há um sensor de efeito Hall, contabilizando, aproximadamente, 4.5 pulsos elétricos por segundo de líquido passado na razão litros/minuto, dividindo o número total de pulsos registrados por 4.5 teremos a quantidade total de líquido passando pelo sensor por minuto (Sanjeev, 2018). Logo, o cálculo para se encontrar a vazão de litros por minuto deve ser o seguinte:

\section{vazão de litros por minuto = número de pulsos contados $/ 4.5$}

Adicionalmente, a Plataforma WACOP define o Módulo de Sumarização, uma API REST responsável por receber os dados vindos do Repositório Intermediário e armazenar os dados previamente sumarizados a fim de facilitar as análises previstas no módulo de gerenciamento, desenvolvida utilizando Java e Spring Boot.

O Módulo Sumarizador conta com um agendador nativo do Spring, responsável por enviar, a cada 60 segundos, os dados do Repositório Intermediário (o Blynk), para o Repositório Central da Plataforma. Este repositório central utiliza como base o MongoDB ${ }^{2}$. Devido sua natureza de trabalhar com dados estruturados em formato de documentos e com alta performance, disponibilidade e escalabilidade para o volume de dados previsto para análises similares a esta de consumo de água.

Esta plataforma também prevê um Módulo de Relatórios e Gerenciamento, o qual permite ao usuário criar e visualizar gráficos de acordo com as informações inseridas no banco de dados em diversos períodos, sendo eles por dia, mês, ano, bem como dados near real-time do consumo. Isso será de extrema utilidade, uma vez que os próprios consumidores do produto poderão criar dashboards de acordo com o tipo e visão que preferirem.

A Arquitetura Proposta foi testada inicialmente através da coleta de dados do fluxo de água (flowRate) em alguns focos em uma residência. Cada foco foi identificado unicamente de forma a coletar e permitir análises individuais. Foram utilizados como atributos identificadores em cada foco o nome do usuário do sistema

\footnotetext{
${ }^{1}$ https://blynk.io/

${ }^{2}$ https://www.mongodb.com/
} 
(userID), MAC Address de cada sensor responsável pelo fluxo de água (deviceID), descrições informativas dos dispositivos (description), e da data e hora da coleta dos dados (timestamp), representando estes dados em Documento do MongoDB conforme a Figura 2.

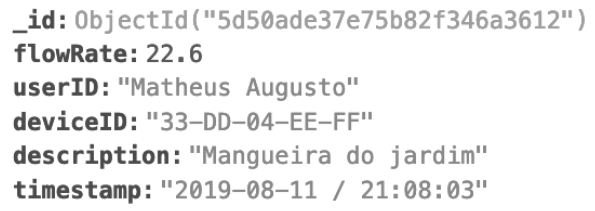

Figura 2. Imagem de um dos Documentos inseridos no MongoDB contendo valores de uma medição de vazão de água

O Módulo de Relatórios da Plataforma WACOP permite uma análise ampla do consumo de água por dispositivo. A Figura 3 apresenta alguns tipos de gráficos que podem ser gerados a partir do total de consumo de água por dispositivo através dos dados coletados em tempo real dos sensores IoT.
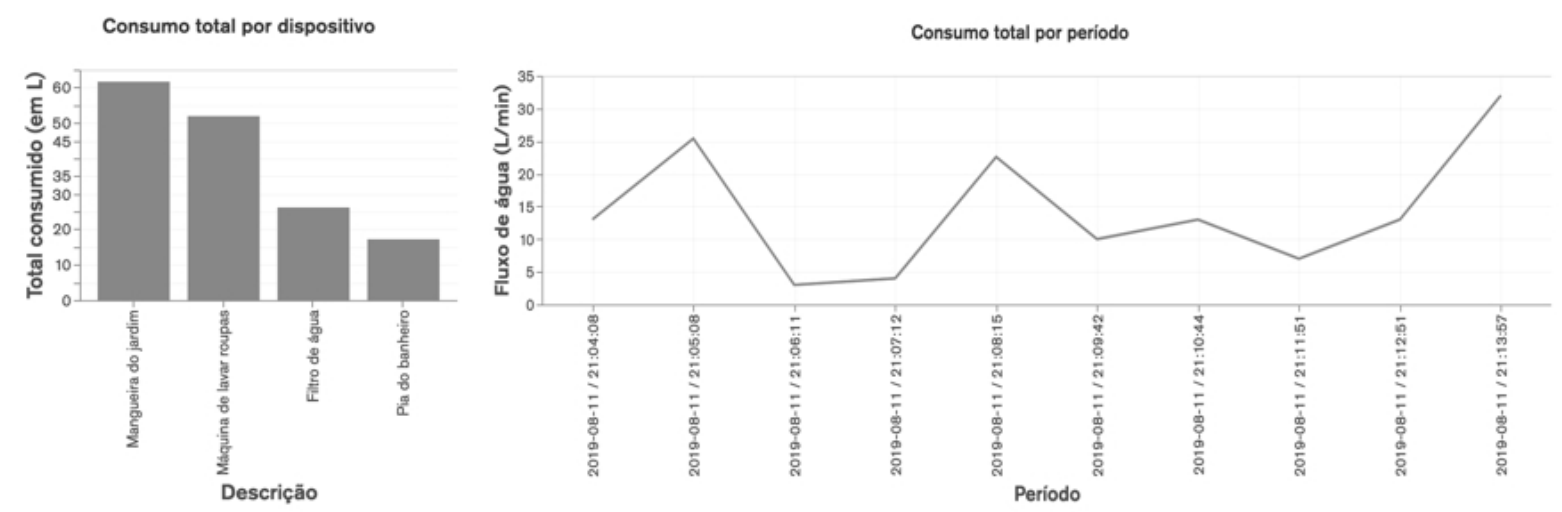

Figura 3. Gráficos representando a quantidade de água coletada por diversos sensores de fluxo de água em tempo real

A análise do gráfico à esquerda permite observar um maior consumo de água no dispositivo acoplado à mangueira do jardim, alcançando a marca dos 60 litros, enquanto que o sensor acoplado à máquina de lavar roupas identificou cerca de 50 litros no total. O filtro de água e pia do banheiro apresentaram um menor índice de utilização de água, chegando a aproximadamente 25 litros e 17 litros, respectivamente, durante o período analisado. O gráfico mais à direita mostra o total de água consumida a cada minuto, apresentando que houve uma grande oscilação geral, cujo índice máximo coletado foi de aproximadamente 33 litros, enquanto que o mínimo foi de 4 litros.

\section{CONCLUSÃO}

Na Europa, 75\% da população já vive em áreas urbanas, este número tem previsão de chegar em $80 \%$ até 2020 (Albino et. al, 2015). A partir desse conhecimento, é essencial que, para promover o gerenciamento inteligente dos recursos naturais já disponíveis, as cidades e seus habitantes comecem a se preocupar com como esses recursos são utilizados, e buscar formas eficientes de utilizá-los.

Os resultados obtidos pelo uso da plataforma WACOP, após a realização das coletas descritas acima, foram satisfatórios e atenderam o que se esperava alcançar. Os usuários poderiam com facilidade acessar seus dados de consumo, podendo alterar o tipo de dashboard disposto, com filtros de período, usuário e descrição do dispositivo, possibilitando uma melhor análise de consumo de água de acordo com a necessidade.

A plataforma proposta neste artigo permite um entendimento do consumo do recurso natural de uma maneira mais completa para os usuários em diferentes níveis (governamental, habitacional e para o cidadão). Sua utilização visa conscientizar cidadãos, estimulando o menor desperdício em suas residências, tendo em vista que também serão apresentadas comparações de consumo de acordo com localidade geográfica e estação do ano de onde aquele indivíduo reside. 
Portanto, as vantagens trazidas por este projeto são agilidade e controle do consumo de água em residências e expansível a condomínios. A Plataforma WACOP possibilita aos cidadãos monitorarem, de maneira simples, rápida e eficaz, a quantidade de litros de água consumidos após tomar um banho, lavar as roupas, escovar os dentes ou qualquer outra atividade cotidiana que a utilize, resultando não apenas em um menor custo mensal para o usuário, como também ajudando o meio ambiente através do uso inteligente de seus recursos.

Como propostas futuras para este projeto, está a de adotar uma forma de não apenas disponibilizar os dados coletados, como também notificar o consumidor sobre um possível vazamento através da análise de padrões do consumo de água, identificando ainda o local do vazamento, e assim minimizar os impactos causados pelo alto consumo. Adicionalmente, é proposto como futuro desenvolvimento, a implementação de uma aplicação mobile para acesso aos dados coletados de forma mais simplificada.

Por fim, fazer com que o Módulo de Relatórios e Gerenciamento disponha de sugestões de consumo por residência ou condomínios, de acordo com cadastros e configurações previamente estabelecidas. Os parâmetros utilizados para sugestões de consumo devem ser obtidos através de dados como estação do ano, localidade geográfica e histórico anual de chuvas da região, compondo assim as análises e relatórios disponibilizados na plataforma, dando uma visão eficiente e detalhada do consumo de água da residência.

\section{REFERÊNCIAS}

Albino, Vito et. al, 2015. Smart Cities: Definitions, Dimensions, Performance, and Initiatives. Journal of Urban Technology, Vol. 22, No. 1, pp 3-21.

Almirall, E. et. al, 2013. A Smart City Initiative: The Case of Barcelona. Journal of the Knowledge Economy, Vol. 4, No. 2, pp 135-148.

Calvillo, C.F. et. al, 2016. Energy management and planning in smart cities. Renewable and Sustainable Energy Reviews, Vol. 55, pp 273-287.

Domoney, Frank W., et. al 2015. Smart Cities Solutions to Water Management using Self-Powered, Low-Cost, Water Sensors and Apache Spark Data Aggregation. Colchester, United Kingdom.

Giffinger, Rudolf et. al, 2007. Smart cities: Ranking of European medium-sized cities. Vienna University of Technology, Viena, Austria.

Guillemin, P. e Friess, P., 2009. Internet of things strategic research roadmap. The Cluster of European Research Projects.

Kon, Fabio e Santana, Eduardo, 2017. Computação aplicada a Cidades Inteligentes: Como dados, serviços e aplicações podem melhorar a qualidade de vidas cidades. Sociedade Brasileira de Computação, Porto Alegre, Brasil.

Liu, P. e Peng, Z., 2014. China's Smart Cities Pilots: A Progress Report. IEEE Computer Society Digital Library.

Lombardi, P. et. al, 2012. Modelling the Smart City Performance. Innovation: The European Journal of Social Science Research. Vol. 25, No. 2, pp 137-149.

Mohanty, Saraju et. al, 2016. Everything You wanted to Know about Smart Cities: The Internet of Things is the backbone. IEEE Consumer Electronics Magazine, Vol. 5, No. 3, pp 60-70.

ONU, 2009. Urban and Rural Areas 2009. United Nations, Department of Economic and Social Affairs, New York, USA.

Patidar, Shyam et.al, 2012. A Survey Paper on Cloud Computing. Proceedings of Second International Conference on Advanced Computing \& Communication Techonologies, Vol., No., pp 394-398.

Perera, Charith et. al, 2014. Sensing as a Service Model for Smart Cities Supported by Internet of Things. Research School of Computer Science, Canberra, Australia.

Procon SP. Qual o consumo médio mensal de água? São Paulo, Brasil.

Sanjeev, Arvind, 2018. How to Interface an Arduino with a Flow Rate Sensor to Measure Liquid: Make an Arduino flow rate sensor to measure water flow for a variety of applications. EETech Media, Boise, USA. 